\title{
Study of antibiotic resistance in patients of enteric perforation in a tertiary care hospital.
}

1. MBBS

Post Graduate Resident General

Surgery

Nishtar Hospital Multan.

2. MBBS, FRCS, FCPS

Assistant Professor Surgery

Nishtar Medical University, Multan.

3. MBBS

Post Graduate Resident Nishtar Hospital Multan.

4. MBBS

Post Graduate Resident Nishtar Hospital, Multan

5. MBBS

Post Graduate Resident

Wolver Hampton Trust Hospital

6. MBBS

Post Graduate Resident

CMH Multan.

\section{Correspondence Address:}

Dr. Muhammad Farrukh Aftab

Department of Surgery

Nishtar Medical University, Multan. imranmerani247@gmail.com

Article received on:

19/05/2020

Accepted for publication:

$19 / 08 / 2020$
Talha Kareem ${ }^{1}$, Muhammad Farrukh Aftab², Junaid Hashmi ${ }^{3}$, Waqas Anjum $^{4}$, Hasaan Rafique ${ }^{5}$, Ali Rabbani ${ }^{6}$

ABSTRACT... Objective: To study the clinical outcomes and determine the antibiotic resistance in patients of enteric perforation in a tertiary care hospital. Study Design: Cross Sectional study. Setting: Department of General Surgery at Nishtar Hospital, Multan. Period: May 2017 to December 2019. Material \& Methods: Sample size was calculated to be 97 with a confidence level of $95 \%$ and margin of error of $10 \%$. We collected data from 100 patients. All the surgeries were done by the same team of consultant surgeons. The abdomen was closed by the same surgical team using the same surgical technique to avoid any bias. All the continuous variables were reported as mean \pm standard deviation. The antibiotic resistance was compared with the number of patients with wound infections, burst abdomen, ICU admissions and number of in-hospital stay days. Categorical variables were compared using Chi square test for Independence. The number of in-hospital stay days were compared using Student t-test. The results were compiled using SPSS version 20. Results: Our result indicated that a patient had a greater chance of having a burst abdomen and wound infection if there was resistance to ciprofloxacin or ceftriaxone. Similar results were obtained for ICU admissions. There was a significantly longer in-hospital stay observed for patients who were resistant to the standard regimen of Ceftriaxone. Conclusion: It seems only rationale that the patients susceptible should be treated with Imipinem for 2 weeks as the empirical therapy rather than the standard empirical therapy of ceftriaxone and ciprofloxacin. The blood cultures can be sent before starting Imipinem and treatment management plan changed if the culture results are sensitive for the empiric antibiotics.

Key words: $\quad$ Antibiotic Resistance, Enteric Perforation, Infectious Diseases.

Article Citation: Kareem T, Aftab MF, Hashmi J, Anjum W, Rafique H, Rabbani A. Study of antibiotic resistance in patients of enteric perforation in a tertiary care hospital. Professional Med J 2021; 28(2):141-146.

https://doi.org/10.29309/TPMJ/2021.28.02.4901

\section{INTRODUCTION}

Typhoid fever has been deemed as the most prevalent bloodstream infection in the South Asian region. ${ }^{1,2}$ The disease is caused by Salmonella enterica typhi and Paratyphi. A. Regardless of the measures to control the disease, this has posed as a burden on public health in South Asia. Some seven million people are estimated to be effected by this calamity every year with 75000 losing the battle; though this figure is thought to be an understatement, mainly due to limited population surveillance systems. ${ }^{3-5}$ Patients in surgical department usually present with the complications of typhoid fever; intestinal perforation being the most sinister of the lot. It usually occurs in the third week of infection and may require resection or exteriorization. ${ }^{6,7}$

Multiple drug resistance has been a major problem in the treatment of Typhoid and other infectious diseases. Initially Chloramphenicol was used for the treatment of typhoid fever. It had a success in the beginning but the cases of resistance started to emerge in 1960s and 1970s. ${ }^{8-10}$ Shortly after, in 1972 there were reports of multiple drug resistance typhoid fever. They weren't just resistant to chloramphenicol but also showed resistance against ampicillin and trimethoprim-sulfamethoxazole. ${ }^{11-13}$ In 2017, certain reports have suggested the presence of strains of Salmonella typhi in Sindh, Pakistan, that are not only resistant to the first line drugs 
used against typhoid fever but stand tall against the likes of fluoroquinolones and third generation cephalosporins. ${ }^{14-16}$ Hence called XDR (extended drug resistance) Salmonella typhi.

\section{OBJECTIVE}

To study the clinical outcomes and determine the antibiotic resistance in patients of enteric perforation in a tertiary care hospital. The sampling technique used was non probability consecutive sampling. The patients included were the ones who have perforation due to typhoid fever.

Cross sectional study. This study was conducted at General Surgery Department of Nishtar Hospital, Multan. From May 2017 to December 2019. This was a cross sectional study.

\section{MATERIAL \& METHODS}

Approval from ethical review board was obtained. The sampling technique used was non probability consecutive sampling. The patients included were the ones who have perforation due to typhoid fever. Patients of all ages and both genders were included. Patients who had been taking antibiotics for the fever or the ones having perforation for any other case were excluded. Sensitivity was checked was for Ampicillin, Trimethoprim Sulfamethoxazole, Third generation cephalosporins, Flouroquinolones and Meropenems.

Sample size was calculated to be 97 with a confidence level of $95 \%$ and margin of error of $10 \%$. We collected data from 100 patients. A written informed consent was taken from all patients before surgery.

Enteric perforation was defined as hole in the anti-mesenteric part of the wall of distal ileum. Symptoms include severe abdominal pain and tenderness. Diagnosis of perforated ileum will be made using Plain supine x-rays of the abdomen (presence of air in the abdominal cavity on x-rays) along with the presenting symptoms (severe abdominal pain) and physical examination (presence of abdominal distension and tenderness) of the patient. ${ }^{17-19}$
All the surgeries were done by the same team of consultant surgeons. The abdomen was closed by the same surgical team using the same surgical technique to avoid any bias. I (the investigator) was assistant in all procedure. Patients follow-up was done after 1 week, 2 weeks and one month of discharge. All the gathered information was noted on a proforma.

Antibiotic resistance was checked on samples of blood culture which will be taken before the first dose of antibiotic. Patients were treated with empirical therapy of ceftriaxone and ciprofloxacin ${ }^{20}$ until the results of sensitivity were received. The blood sample were sent to the Nishtar Pathology laboratory.

Patients were closely followed up for burst abdomen while they were admitted in the ward and for wound infection according to the Southampton scoring system. ${ }^{21,22}$ The wound infection was observed subsequently on follow up visits as well. Patient was asked to have a follow up check-up weekly for one month after discharge.

The burst abdomen includes the patients in which the continuation of the disease process led to the giving way of the abdominal layers or a perforation proximal to the ileostomy lead to re exploration of the patient

All the continuous variables were reported as mean \pm standard deviation. The antibiotic resistance was compared with the number of patients with wound infections, burst abdomen, ICU admissions and number of in-hospital stay days. Categorical variables were compared using Chi square test for Independence. The number of in-hospital stay days were compared using Student t-test. The results were compiled using SPSS version 20 .

\section{RESULTS}

The prevalence of drug resistance for different groups is demonstrated in Figure-1.

To assess the length of hospital-stay in patients, Student $t$ test was applied. There was a significantly longer in-hospital stay observed 
for patients who were resistant to the standard regimen of Ceftriaxone.

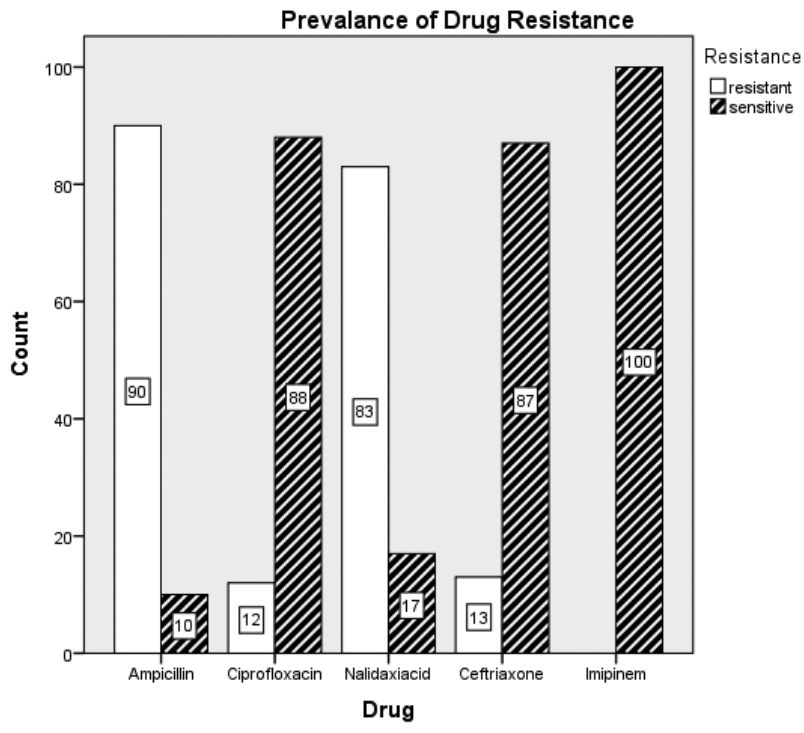

Our result indicated that a patient had a greater chance of having a burst abdomen if there was resistance to ciprofloxacin or ceftriaxone.
Similarly, the rate of wound infection, as assessed by Southampton Scoring ${ }^{23}$, was higher in patients with ciprofloxacin \& ceftriaxone resistance.

Similar results were obtained for the rate of surgical wound infection \& the ICU admissions.

ICU admissions were also comparable to these aforementioned results. The fact that resistant patients failed to respond to the empiric therapy, led to multiple complications of typhoid fever. These complications also included re exploration due to another perforation in the terminal ileum.

\section{DISCUSSION}

Pakistan is one of the few regions of the world where the extensively drug resistant Salmonella Typhihas beenreported. ${ }^{23,24}$

\begin{tabular}{|l|c|c|c|c|}
\hline \multicolumn{1}{|c|}{ Drug } & Resistance & N & $\begin{array}{c}\text { Hospital Stay (days) } \\
\text { Mean } \pm \text { SD }\end{array}$ & P-Value \\
\hline \multirow{2}{*}{ Ampicillin } & resistant & 90 & $8.69 \pm 6.81$ & 0.93 \\
\hline \multirow{2}{*}{ Ciprofloxacin } & sensitive & 10 & $8.90 \pm 7.74$ & 0.17 \\
\cline { 2 - 5 } & resistant & 12 & $11.25 \pm 6.97$ & 0.42 \\
\hline \multirow{2}{*}{ Nalidaxiacid } & sensitive & 88 & $8.36 \pm 6.82$ & 0.7 .02 \\
\hline \multirow{2}{*}{ Ceftriaxone } & resistant & 83 & $13.38 \pm 7.65$ & $<0.01$ \\
\hline \multirow{2}{*}{ Imipinem } & sensitive & 17 & $8.01 \pm 6.50$ \\
\hline
\end{tabular}

Table-I. Difference between in-hospital stay days based on drug resistance, checked by student t test

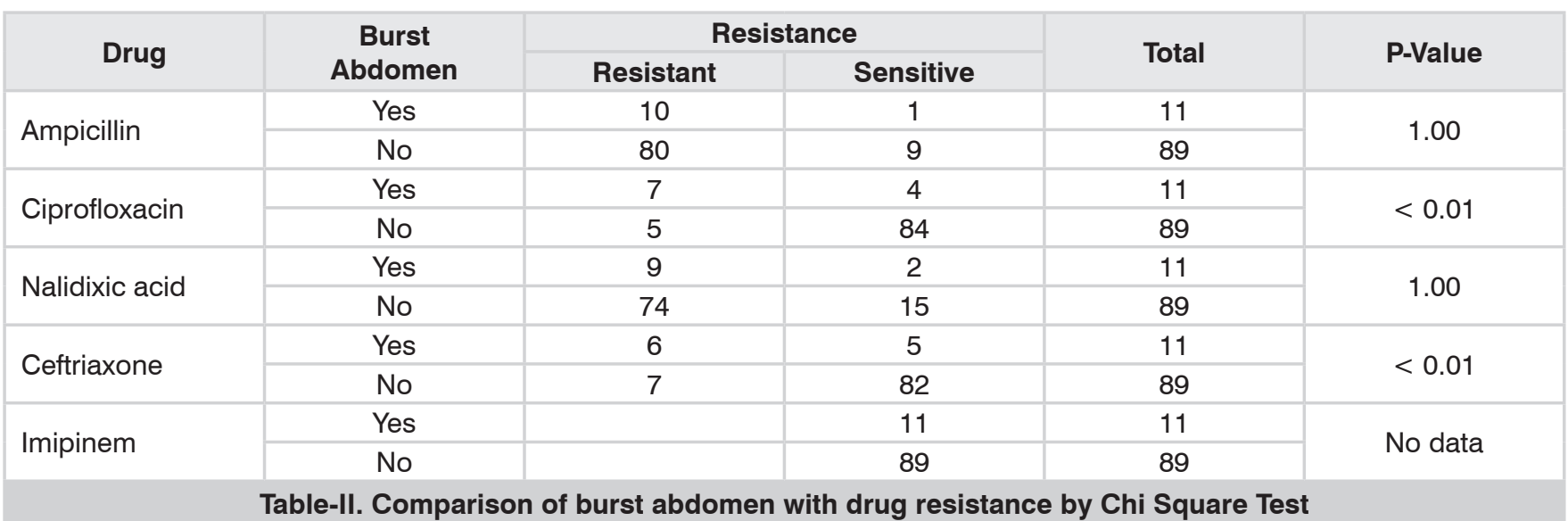




\begin{tabular}{|c|c|c|c|c|c|}
\hline \multicolumn{6}{|c|}{ Drug Resistance vs Wound Infection } \\
\hline \multirow{2}{*}{ Drug } & \multirow{2}{*}{$\begin{array}{l}\text { Wound } \\
\text { Infection }\end{array}$} & \multicolumn{2}{|c|}{ Resistance } & \multirow{2}{*}{ Total } & \multirow{2}{*}{ P-Value } \\
\hline & & Resistant & Sensitive & & \\
\hline \multirow{2}{*}{ Ampicillin } & Yes & 18 & 3 & 21 & \multirow{2}{*}{0.43} \\
\hline & No & 72 & 7 & 79 & \\
\hline \multirow{2}{*}{ Ciprofloxacin } & Yes & 8 & 13 & 21 & \multirow{2}{*}{$<0.01$} \\
\hline & No & 4 & 75 & 79 & \\
\hline \multirow{2}{*}{ Nalidixic acid } & Yes & 18 & 3 & 21 & \multirow{2}{*}{1.0} \\
\hline & No & 65 & 14 & 79 & \\
\hline \multirow{2}{*}{ Ceftriaxone } & Yes & 11 & 10 & 21 & \multirow{2}{*}{$<0.01$} \\
\hline & No & 2 & 77 & 79 & \\
\hline \multirow{2}{*}{ Imipinem } & Yes & & 21 & 21 & \multirow{2}{*}{ No data } \\
\hline & No & & 79 & 79 & \\
\hline
\end{tabular}

Table-III. Comparison of wound Infection with drug resistance by Chi Square Test

\begin{tabular}{|c|c|c|c|c|c|}
\hline \multicolumn{6}{|c|}{ Drug Resistance vs ICU Admissions } \\
\hline \multirow{2}{*}{ Drug } & \multirow{2}{*}{$\begin{array}{c}\text { ICU } \\
\text { Admissions }\end{array}$} & \multicolumn{2}{|c|}{ Resistance } & \multirow{2}{*}{ Total } & \multirow{2}{*}{ P-Value } \\
\hline & & resistant & sensitive & & \\
\hline \multirow{2}{*}{ Ampicillin } & Yes & 8 & 1 & 9 & \multirow{2}{*}{1.00} \\
\hline & No & 82 & 9 & 91 & \\
\hline \multirow{2}{*}{ Ciprofloxacin } & Yes & 5 & 4 & 9 & \multirow{2}{*}{.001} \\
\hline & No & 7 & 84 & 91 & \\
\hline \multirow{2}{*}{ Nalidixic acid } & Yes & 8 & 1 & 9 & \multirow{2}{*}{1.00} \\
\hline & No & 75 & 16 & 91 & \\
\hline \multirow{2}{*}{ Ceftriaxone } & Yes & 5 & 4 & 9 & \multirow{2}{*}{0.002} \\
\hline & No & 8 & 83 & 91 & \\
\hline \multirow{2}{*}{ Imipinem } & Yes & & 9 & 9 & \multirow{2}{*}{ No data } \\
\hline & No & & 91 & 91 & \\
\hline
\end{tabular}

The result of these resistant strains has not only posed a burden on our healthcare system but has made us as clinicians unsure if the empirical therapy that we are going to start a patient is going to get the job done effectively. ${ }^{25}$ The data collected in this research has shown that ampicillin and Nalidixic acid are no longer nearly enough for this battle against the resistant typhoid strains. The worrisome is the fact that resistance seem to be emerging against ciprofloxacin and ceftriaxone as well. The patients with the resistance against the empiric treatment did not only have a longer stay at the hospital but also a greater incidence of burst abdomen and a greater deal of ICU admissions. This problem of typhoid outbreak has been reported in people who have recently travelled to Pakistan. Recommendations have been made that since these strains are susceptible to imipinems and azithromycin, the susceptible patients should be treated with these antibiotics as first line medications rather than the standard empirical treatment. ${ }^{24}$

Not only this but the wound infection rates in the patients who had been infected with resistant strains of Salmonella Typhi were significant as compared with the patients with sensitive strains. The probable reason for this is the initial treatment with the empiric therapy failed to curb the infection and by the time culture results arrived and we started the patients on imipenem, the wound infection had already set in.

\section{CONCLUSION}

Majority of the patients were resistant to ampicillin and nalidixic acid. There is an emergent resistance to ciprofloxacin and ceftriaxone that render patients prone to complications and extended hospital stay. The resistance against imipenem in 
Salmonella typhi is still very low.

Copyright@ 19 Aug, 2020.

\section{REFERENCES}

1. Deen J, Von Seidlein L, Andersen F, Elle N, White NJ, Lubell Y. Community-acquired bacterial bloodstream infections in developing countries in south and southeast Asia: A systematic review. The Lancet infectious diseases. 2012; 12(6):480-7.

2. Ahmed D, Nahid MA, Sami AB, Halim F, Akter N, Sadique $\mathrm{T}$, et al. Bacterial etiology of bloodstream infections and antimicrobial resistance in Dhaka, Bangladesh, 2005-2014. Antimicrobial Resistance \& Infection Control. 2017; 6(1):2.

3. Mogasale V, Maskery B, Ochiai RL, Lee JS, Mogasale VV, Ramani E, et al. Burden of typhoid fever in lowincome and middle-income countries: A systematic, literature-based update with risk-factor adjustment. The Lancet Global health. 2014; 2(10):e570-e80.

4. Barkume C, Date K, Saha SK, Qamar FN, Sur D, Andrews JR, et al. Phase I of the surveillance for enteric fever in Asia Project (SEAP): An overview and lessons learned. The Journal of infectious diseases. 2018; 218(suppl_4):S188-S94.

5. Luby SP. Urban slums: A supportive ecosystem for typhoidal Salmonellae. The Journal of infectious diseases. 2018; 218(suppl_4):S250-S4.

6. Azhar M, Zamir N, Shaikh M. Enteric fever complicated by intestinal perforation in children: A persistent health problem requiring surgical management. Pakistan Journal of Medical Sciences. 2020; 36(5):890.

7. Qureshi SA, Khan Ml, Arbab R. Outcomes of primary repair in typhoid perforation. Annals of PIMS-Shaheed Zulfiqar Ali Bhutto Medical University. 2017; 13(4):296300.

8. Murti BR, Rajyalakshmi K, Bhaskaran C. Resistance of salmonella typhi to chloramphenicol: Part IA preliminary report. Journal of clinical pathology. 1962; 15(6):544-8.

9. Olgemoeller F, Waluza JJ, Zeka D, Gauld JS, Diggle PJ, Read JM, et al. Intestinal perforations associated with a high mortality and frequent complications during an epidemic of multidrug-resistant typhoid fever in Blantyre, Malawi. Clinical Infectious Diseases. 2020; 71(Supplement_2):S96-S101.

10. Dyson ZA, Klemm EJ, Palmer S, Dougan G. Antibiotic resistance and typhoid. Clinical Infectious Diseases. 2019; 68(Supplement_2):S165-S70.
11. Olarte J, Galindo E. Salmonella typhi resistant to chloramphenicol, ampicillin, and other antimicrobial agents: strains isolated during an extensive typhoid fever epidemic in Mexico. Antimicrobial agents and chemotherapy. 1973; 4(6):597-601.

12. Saha S, Sajib MSI, Garrett D, Qamar FN. Antimicrobial resistance in typhoidal salmonella: Around the world in 3 days. Clinical Infectious Diseases. 2020; 71(Supplement_2):S91-S5.

13. Yang Y-A, Chong A, Song J. Why is eradicating typhoid fever so challenging: Implications for vaccine and therapeutic design. Vaccines. 2018; 6(3):45.

14. Akram J, Khan AS, Khan HA, Gilani SA, Akram SJ, Ahmad FJ, et al. Extensively Drug-Resistant (XDR) Typhoid: Evolution, Prevention, and Its Management. BioMed Research International. 2020; 2020.

15. Khan EA. XDR Typhoid: The problem and its solution. Journal of Ayub Medical College Abbottabad. 2019; $31(2): 139-40$.

16. Cohen J. 'Frightening'typhoid fever outbreak spreads in Pakistan. American Association for the Advancement of Science; 2018.

17. Somani K, Vashistha R, Datey S, Gurjar A, Patel A. An observational study of clinical profile and management of non-traumatic small bowel perforation at tertiary care centre. Journal of Evolution of Medical and Dental Sciences. 2018; 7(32):3581-5.

18. Malhotra MK, Singal R, Chowdhary K, Sharma RG, Sharma S, Dhankhar A. Spectrum of perforation peritonitis in a Rural Medical College. Bangladesh Journal of Medical Science. 2016; 15(1):70-3.

19. Marwah S. Small bowel perforations. Abdominal Sepsis: Springer; 2018. p. 105-62.

20. Veeraraghavan B, Pragasam AK, Bakthavatchalam YD, Ralph R. Typhoid fever: Issues in laboratory detection, treatment options \& concerns in management in developing countries. Future science OA. 2018; 4(6):FSO312.

21. Akhter MSJ, Verma R, Madhukar KP, Vaishampayan AR, Unadkat $P$. Incidence of surgical site infection in postoperative patients at a tertiary care centre in India. Journal of Wound Care. 2016; 25(4):210-7.

22. Hussain A, Tassadaq N. General health status in patients of adhesive capsulitis visiting rehabilitation department of Fauji Foundation Hospital Rawalpindi, Pakistan: General Health Status in Adhesive Capsulitis. Int J Front Sci [Internet]. 2020; 4(2). Available from: https://publie.frontierscienceassociates. com/index.php/tijfs/article/view/185. 
23. Klemm EJ, Shakoor S, Page AJ, Qamar FN, Judge K, Saeed DK, et al. Emergence of an extensively drug-resistant Salmonella enterica serovar Typhi clone harboring a promiscuous plasmid encoding resistance to fluoroquinolones and third-generation cephalosporins. MBio. 2018; 9(1):e00105-18.

24. Bashir N, Ahmed A, Raza H, Imran M. Relationship between the Susceptibility of Salmonella.Typhi to Ciprofloxacin, Ofloxacin and their respective Susceptibility To Nalidixic Acid. Int J Front Sci [Internet]. 2018; 2(2):33-44. Available from: https:// publie.frontierscienceassociates.com/index.php/tijfs/ article/view/40.
25. Wong W, Al Rawahi H, Patel S, Yau Y, Eshaghi A, Zittermann $S$, et al. The first Canadian pediatric case of extensively drug-resistant Salmonella Typhi originating from an outbreak in Pakistan and its implication for empiric antimicrobial choices. IDCases. 2019; 15:e00492.

AUTHORSHIP AND CONTRIBUTION DECLARATION
\begin{tabular}{|c|l|l|}
\hline Sr. \# & Author(s) Full Name & \multicolumn{1}{c}{ Contribution to the paper } \\
\hline 1 & Talha Kareem & Conceive idea, Proofeading. \\
\hline 2 & M. Farrukh Aftab & Data analysis, Result writing. \\
\hline 3 & Junaid Hashmi & Manuscript writing. \\
\hline 4 & Waqas Anjum & Data collection and Data entry. \\
\hline 5 & Hasaan Rafique & Data collection and Data entry. \\
\hline
\end{tabular}

